\title{
I limiti della dialisi peritoneale
}

\author{
Nicola Di Paolo ${ }^{1}$, Umberto Buoncristiani ${ }^{2}$ \\ ${ }^{1}$ Nefrologo, Siena \\ ${ }^{2}$ Nefrologo, Perugia
}

\begin{abstract}
The LIMITS OF PERITONEAL DIALYSIS
Abstract. Peritoneal dialysis (PD) failed to meet the expectations not only of nephrologists but also of those companies that invested so much in this field. After 40 years of studies, the main evidences show the little biocompatibility of PD and the unexpected strong reaction of the peritoneum, which tries in every way to defend itself against what it considers an external aggression. Although much has been done to make PD more easily acceptable, this procedure implies a number of frequent complications that urge the nephrologist to use PD only when there are absolute indications for intracorporeal treatment or contraindications to extracorporeal dialysis, thus excluding, for the moment, PD as a treatment of first choice.
\end{abstract}

Key words: Peritoneal dialysis, Biocompatibility, Mesothelial cell

Conflict of interest: None.

Financial support: None.

Accettato: 28 Luglio 2013

Oggi, a distanza di 35 anni dall'avvento della CAPD, siamo a chiederci quali sono le ragioni per le quali la dialisi peritoneale continua a essere un trattamento di seconda scelta rispetto alla dialisi extracorporea, rimanendo al di sotto del $10 \%$ di tutta la popolazione dialitica mondiale con un trend negativo nei paesi industrializzati.

A nostro parere, non è difficile rispondere a questa domanda; basta convincersi che la dialisi peritoneale, contrariamente a quanto supposto e sostenuto per tanto tempo, è certamente meno biocompatibile di quella extracorporea.

All'inizio eravamo tutti convinti che la dialisi intracorporea, effettuata all'interno dell'addome, fosse il trattamento depurativo più consono all'organismo umano, partendo dal presupposto che il mesotelio che ricopre il peritoneo era stato per secoli ritenuto un semplice epitelio piatto, guarnito di microvilli che aumentavano enormemente la superficie di contatto e, quindi, di scambio con le soluzioni di dialisi peritoneale, che sembravano ben tollerate e che potevano rimanere in addome senza alcuna interruzione e senza arrecare danni.

$\mathrm{Ci}$ sono voluti anni e anni di ricerche, prima condotte solo da nefrologi e, in seguito, da morfologi, biologi e chirurghi, per capire che le cellule mesoteliali erano ben differenti da quello che si era supposto fino ad allora. Dapprima si evidenziò che erano cellule secernenti in grandi quantità una miscela di fosfolipidi, in massima parte costituita da fosfatidilcolina, dando origine a una sostanza almeno 100 volte più viscosa dell'olio d'oliva e che permette un perfetto scivolamento degli organi interni, rivestiti dalle sierose, assicurandone la funzionalità. Purtroppo, si evidenziò che la dialisi peritoneale causava una perdita enorme di questa miscela fosfolipidica.

Questo fu solo l'inizio; da allora, pian piano, si scoprì che la cellula mesoteliale secerneva una quantità di sostanze tra le più varie e le più impensate, con azione fibrinolitica, antinfettiva, antinfiammatoria e antitumorale, che produceva fattori di crescita, stimolatori della neoangiogenesi, interleuchine e molti altri ancora e che tutte queste sostanze venivano continuamente sottratte con la dialisi peritoneale, costringendo il mesotelio, per un meccanismo di feed-back, a produrne il più possibile.

Sono stati molti i nefrologi che, supportati dalle aziende produttrici di soluzioni di dialisi peritoneale, iniziarono a intraprendere studi complessi sulla biocompatibilità delle soluzioni e dei cateteri peritoneali. Tanto per citarne un paio, ricorderemo l'importanza della sostituzione del tampone acetato prima con il lattato e poi con il bicarbonato, evitando, così, la precoce e grave comparsa della peritonite sclerosante, nonché i numerosi tentativi per cercare di sostituire il glucosio, sostanza dai potenti poteri osmotici, ma causa di serie e grossolane alterazioni vascolari simildiabetiche. Alcune soluzioni con aminoacidi e icodestrina hanno solo in piccola parte sostituito il glucosio. Le più avanzate realizzazioni sono soluzioni a $\mathrm{pH}$ neutro contenenti minime quantità di GDP, ma ancora tutte lontane da una biocompatibilità accettabile. Del tutto recentemente è apparsa una pubblicazione che propone una soluzione contenente L carnitina, che verrà sperimentata nell'uomo.

Difficile, comunque, pensare a una risoluzione del problema. Infatti, fino a poco tempo fa, non ci si rendeva ancora minimamente conto delle potenzialità delle cellule mesoteliali e dello stesso peritoneo che, durante la dialisi peritoneale, mettono in atto tutti 


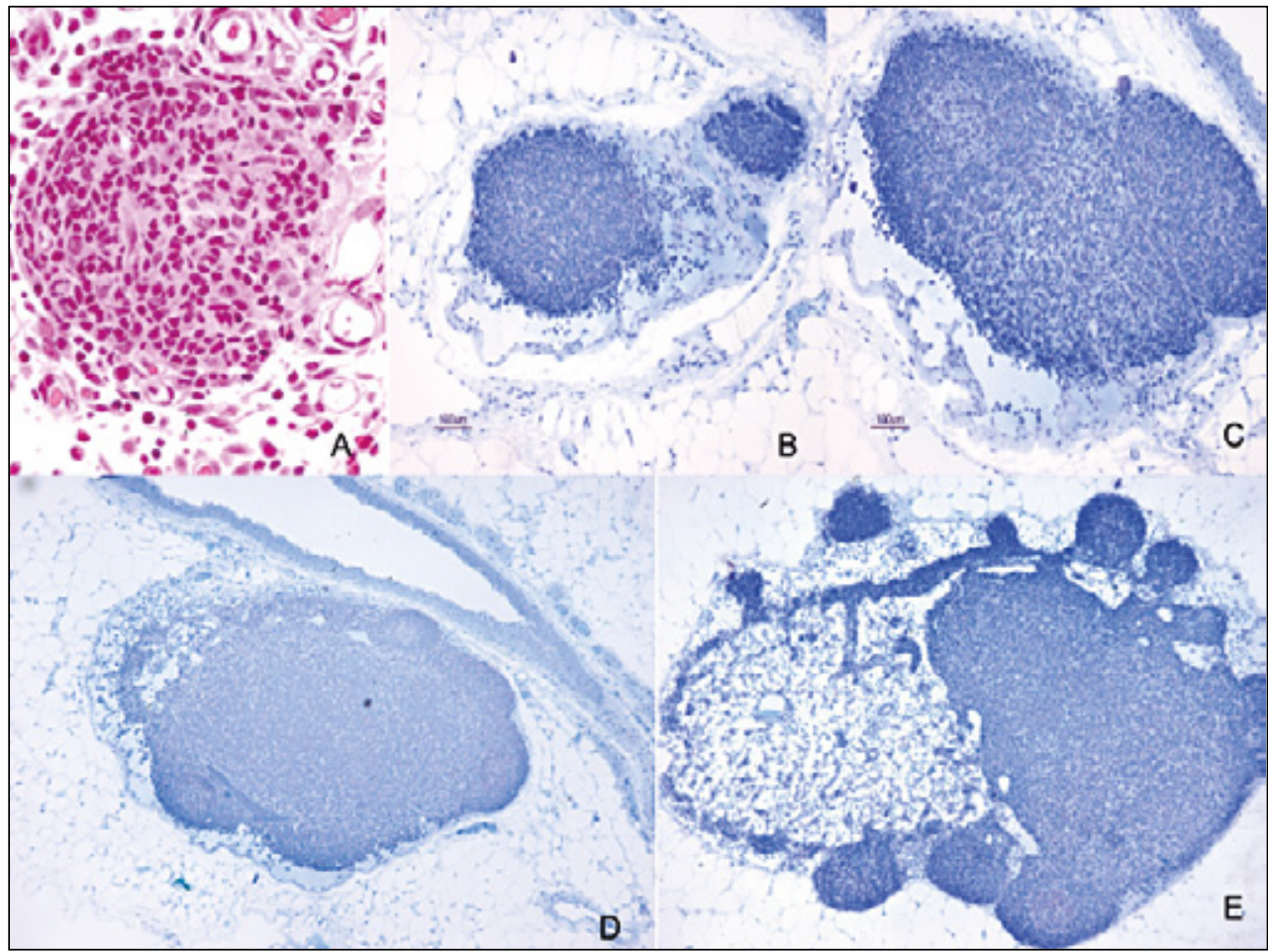

Fig. 1 - Evoluzione di un singolo "milky spot" da semplice organo microscopico (A), composto da soli macrofagi disposti attorno a un vaso. Con il tempo, la dialisi peritoneale (B-E) ne provoca la trasformazione in strutture enormemente più grandi, simili a linfonodi, dove i macrofagi mantengono la maggiore rappresentanza.

i meccanismi possibili per difendersi dai corpi e dalle sostanze estranei che vengono messi a contatto con la loro struttura. Il peritoneo, durante la dialisi, non fa che difendersi, cercando di eliminare ciò che di estraneo entra in contatto con lui. Il peritoneo così come la pleura e il pericardio sono così importanti dal punto di vista della loro fisiologia che i sistemi di difesa e di rigetto sono più complicati di ciò che si poteva pensare un tempo. In compenso, gli studi di morfologia e di fisiologia delle sierose si sono man mano ampliati, portando a risultati impensabili.

La coltura delle cellule mesoteliali in particolare ha potuto, con il tempo, dimostrare che queste cellule non solo secernono una quantità enorme di sostanze, ma sono anche capaci di trasformarsi in fibroblasti, osteoblasti, cellule midollari, cellule epiteliali, macrofagi e altro ancora, rendendole molto simili a una cellula staminale. Gli studi morfologici hanno, poi, dimostrato che l'omento, ritenuto una semplice struttura formata da due fogli di cellule mesoteliali contenenti all'interno grasso e vasi, è, in realtà, un insieme di elementi più eterogeneo, ospitando, nel suo complesso, corpuscoli evidenziabili facilmente a occhio nudo o, meglio, colorandoli con inchiostro di china, costituiti da ammassi di macrofagi attorno a un vaso. Lunghi filamenti collegano tra loro questi corpuscoli nominati " milky spots", creando una rete multiforme la cui funzione è ancora lungi dall'essere spiegata. È verosimile, tuttavia, supporre che questi piccoli organi facciano parte del complicato sistema difensivo delle sierose dell'organismo e che giochino un ruolo importante nella dialisi peritoneale (Fig. 1).

C'è da sottolineare, d'altra parte, che le soluzioni di dialisi e i cateteri sono, negli anni, migliorati nella loro composizione, ma che sono lungi ancora dall'essere sufficientemente biocompatibili. Rimane il fatto che, anche se, al momento attuale, sono in corso interessanti studi tendenti a rendere biocompatibile la dialisi peritoneale, per ora siamo sempre distanti da quel traguardo.

La dialisi extracorporea, pur essendo anche lei scarsamente biocompatibile, viene applicata 12 ore a settimana contro le 168 ore della dialisi peritoneale. Basterebbe questo dato, senza stare a elencare i grandi progressi raggiunti nella produzione di membrane, macchine e soluzioni per la dialisi extracorporea, a rendere la peritoneale molto meno biocompatibile della dialisi extracorporea stessa. Se è vero, quindi, che la dialisi peritoneale è estremamente positiva dal punto di vista depurativo per la sua continuità e, quindi, per la sua fisiologia, è anche evidente che il danno è continuo; fisiologia e biocompatibilità non vanno d'accordo in questo contesto e, per ora, non si vedono vie di uscita dal presente stato.

Quali sono gli effetti della scarsa biocompatibilità? La sclerosi, la non funzionalità, la facilità a contrarre infezioni che provocano, a loro volta, danni gravissimi e spesso irreparabili al peritoneo, l'estrusione delle cuffie, il dolore addominale, i disturbi digestivi e le alterazioni metaboliche sono tutti aspetti riferibili al potente rigetto del peritoneo verso sostanze estranee. Questa capacità organica di difesa, sviluppata in centinaia di migliaia di anni, è ancora lungi dall'essere completamente chiarita.

Trentacinque anni di studi, se, da un lato, hanno portato i chirurghi a intuire la possibilità di utilizzare il mesotelio a scopi terapeutici (vedi le applicazioni di trapianto e impianto di cellule mesoteliali che si comportano in maniera simile alle staminali), hanno, d'altra parte, scoraggiato i nefrologi a usare in modo indiscriminato la dialisi peritoneale, cercando di applicarla dove veramente è utile, senza forzare il suo impiego come trattamento di prima scelta nella sostituzione della funzione renale. 


\section{Riassunto}

La dialisi peritoneale ha disatteso le aspettative dei nefrologi e di quella parte dell'industria che tanto ha investito in questo campo. Dopo 40 anni di studi, l'evidenza principale è stata quella della scarsa biocompatibilità della DP e dell'inaspettata potente reazione di difesa del peritoneo, che cerca in tutti i modi di difendersi da quella che ritiene una vera e propria aggressione esterna. Anche se molto è stato fatto per rendere più accettabile il trattamento, questo è ancora oggetto di frequenti complicazioni, che devono indirizzare il nefrologo a impiegare la DP solo dove esistono indicazioni assolute o controindicazioni alla dialisi extracorporea, tralasciando, per ora, la DP come trattamento di prima scelta.

Parole chiave: Dialisi peritoneale, Biocompatibilità, Cellula mesoteliale
Dichiarazione di conflitto di interessi: Gli Autori dichiarano di non avere conflitto di interessi.

Contributi economici degli autori: Gli Autori dichiarano di non aver ricevuto sponsorizzazioni economiche per la preparazione dell'articolo.

Indirizzo degli Autori:

Prof. Nicola Di Paolo

Strada di Vignano 2

53100 Siena

n.dipaolo@libero.it

\section{Bibliografia}

1. Devuyst O, Margetts PJ, Topley N. The pathophysiology of the peritoneal membrane. J Am Soc Nephrol 2010; 21 (7): 1077-85.

2. Gotloib L, Gotloib LC, Khrizman V. The use of peritoneal mesothelium as a potential source of adult stem cells. Int J Artif Organs 2007; 30 (6): 501-12.
3. Diaz-Buxo JA, Gotloib L. Agents that modulate peritoneal membrane structure and function. Perit Dial Int 2007; 27 (1): 16-30.

4. Mutsaers SE, Di Paolo N. Future directions in mesothelial transplantation research. Int J Artif Organs 2007; 30 (6): 557-61.

5. Di Paolo N, Sacchi G. Atlas of peritoneal histology. Perit Dial Int 2000; 20 (Suppl. 3): S5-96. 